\title{
Development of Radio Frequency Heating Technology for Shale Oil Extraction
}

\author{
Yi Pan, Can Chen, Shuangchun Yang, Guiyang Ma \\ Liaoning Shihua University, Fushun, China \\ Email: panyi_bj@126.com
}

Received February 20, 2012; revised March 18, 2012; accepted March 22, 2012

\begin{abstract}
With the shortage of energy and the rise of crude oil in the world, the development of oil shale is gaining more attention globally. To solve the problem of traditional heat conduction with low efficiency and high cost for oil shale, a new technology called radio frequency (RF) heating (microwave heating) is introduced in this paper. Radio frequency electromagnetic (microwave) can do work directly on medium molecule of oil shale and change into heat energy, the transmission of which allows both inner and outer molecules heating simultaneously without heat conduction. Meanwhile, oil shale is a poor microwave absorbing material and microwave absorbents must be added to reach pyrolysis temperature. By this means, shale oil could be heated at a higher speed and kerogen will gradually be cracked into gas and oil. Then shale oil and gas will flow into the production wells through fractures generated by heating and be pumped up to the surface.
\end{abstract}

Keywords: Oil Shale; Shale Oil; Radio Frequency Heating; Microwave

\section{Introduction}

Oil shale is a significant fossil resource with substantial reserves, which is as high as 400 billion if all be converted into shale oil ,and it is equivalent to 5.4 times of the world's proven crude oil [1,2] .China is one of the richest countries in oil shale resources over the world, with 31.567 billion tons of proven oil shale reserves, which mainly distributes in MaoMing, HuaDian, FuShun, etc., ranking the fourth followed by America, Estonia and Brazil in the world $[3,4]$. As a potential important resource, many of the world's companies have been working on the research and development of the shale oil. In a report of the US Department of Energy, it is confirmed that more than thirty American enterprises actively involved in the project of the oil shale. With the world's energy demands growing, it's worthwhile seeking for the effective development and economic utilization.

Oil shale, which mainly composed of mineral and kerogen, is a kind of low permeability rock. Kerogen is the parent material of oil and natural gas. Although the world has a large oil shale reserves, a large portion of which is buried relatively deep (under $500 \mathrm{~m}$ ). So, the open and tunnel retorting system can't be utilized. Only by means of heating the oil shale formation, the shale oil can be extracted effectively, which is commonly called in-situ retorting technique. Currently, three heating methods have been adopted, namely conduction heating, con- vection heating and radiation heating respectively. Compared with the first two means which need long retorting time and have low extraction rate [5], the microwave heating can greatly make up for the deficiencies.

\section{Microwave Heating of Oil Shale}

\subsection{Priciples}

Microwave has dielectric thermal effect on materials, by which the migration of ion and the rotary of polarity molecules that make the molecules moving. That is, the relatively static transient of polarity molecules changes into dynamic and produces thermal effect by the dipolar high-speed rotating of molecules. The change happens in the inner part of the rolled substance that is internal heating. For oil shale is a poor conductor of heat, the utilization of conduction heating and convection heating will lead to low speed. While the adoption of radio frequency heating overcome the difficulties of heating underground oil shale, which makes the retorting easier and more effectively.

\subsection{Mining Process}

Lay nine wells in the single level oil shale seam, with the thickness of $40 \mathrm{~m}$ and the buried depth of $300 \mathrm{~m}$. Well 4 to well 9 locate on the intersection of squares with the distance of $16 \mathrm{~m}$, showing a positive hexagon grid distri- 
bution. Well 1 to well 3 are heating wells, the distance of which is $3 \mathrm{~m}$ (Ordinarily, the distance between wells is designed as the frequency of wave. The higher the frequency, the shorter the distance will be. The lower the frequency, the longer the distance between wells [6].) and drill each well into seam 10 .Then place a microwave generator in well 2 and radiate microwave to heat oil shale formation as the predetermined direction (See Figures 1 and 2). Detailed steps are introduced as follows:

- Dill pinnate well 1 and 3 in the upper and lower seam of oil shale respectively and inject microwave absorbents, forming symmetrical microwave absorbing surface.

- Drill horizontal well 2 that radiate microwave and perforate 20 holes at the wall of cased hole. Thus two pinnate wells and a horizontal well make a heating well group.

- Drill vertical well 4 to 9 as producing wells.

- Turn on the microwave generator and radiate microwave under the power of $1700 \mathrm{kw}$. Oil and gas will be extracted through producing wells after 60 days of pyrolysis of oil shale formation. To guarantee that the underground oil and gas will flow in gas phase, the power of microwave generator and the closing time of producing wells can be adjusted to control the un-

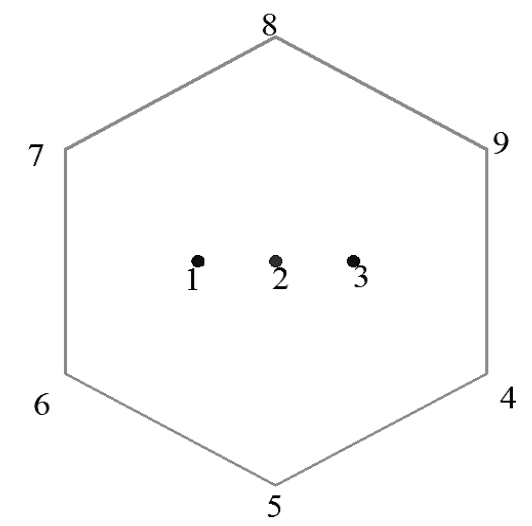

Figure 1. Well network layout of microwave heating.

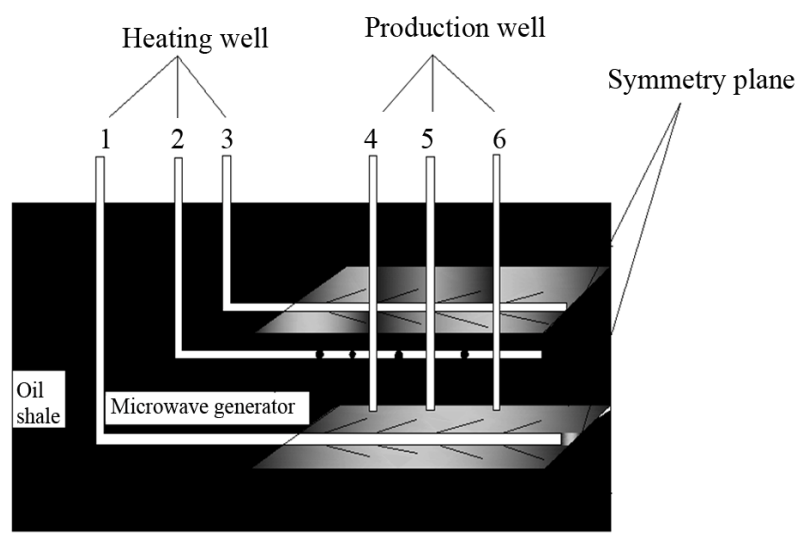

Figure 2. Well network profile of microwave heating. derground pressure and temperature. When fluids exist in gas phase and the temperature of released oil and gas is below 200 centigrade, oil and gas can be continuously released and get separated on the ground.

- Later in the retorting process, turn off the microwave generator and convert pinnate wells and horizontal wells into injection wells when little oil and gas flow out of the producing wells. Inject cold water, drive the residual oil and gas, also it will lower the temperature of subsurface formation. The cold water heated by the subsurface stratum is recovered through other wells, which can be used another well network after disposition [7].

\subsection{Technique Characteristics}

Three ways can be adopted to transfer the heat to oil shale, namely conduction heating, convection heating and radiation heating [8]. Compared with other heating techniques, microwave heating technique has advantages of strong penetration, high heating speed and efficiency, Real-Time heating, integrity, etc.

- The Real-time represents it heats rapidly when taking microwave to heat materials. Microwave radiation promote polarity molecules to move severely, making molecules collide and rub. The heating process takes place in the whole object simultaneously with quickness in temperature rise, temperature uniformity and low temperature gradient.

- The high efficiency of energy consumption mainly reflects that medium materials can absorb microwave and change it into heat energy directly. Microwave heating requires no heat conduction.

- Microwave heating has good selectivity, and it can play different roles in different materials. With turning off the microwave source, microwave energy is unavailable, which can control the temperature of oil shale accurately.

- The crack of oil shale by microwave heating will increase the fractures, which contributes to the transmission of microwave in oil shale, expanding heating area and the discharge.

- Heating shale oil by microwave, heating rate can be easily adjusted. Conventional methods require a quite long time to reach the predetermined temperature and longer cooling time when stop heating. With microwave heating process, the status of oil shale will change as the output power of microwave regulated.

\section{The Present Situation of Radio Frequency Technique}

\subsection{RF/CF Technology Introduction of Raytheon}

As the expert in RF field, Raytheon has made a perfect 
combination of his RF and CF which is from Hyde Park, the other expert in CF field. By this in-situ technology, oil shale is heated by $\mathrm{CF}$, and critical $\mathrm{CO}_{2}$ drives the liquid and gas out (as shown in Figure 3).

\subsubsection{Process Introduction}

$\mathrm{RF} / \mathrm{CF}$ is a patent technology that combined radio frequency heating and critical liquid driving [9]. The oil industry equipment drills and sends radio frequency antenna or transmitter to the oil shale. The ray energy from antenna or transmitter heats the oil shale, and critical $\mathrm{CO}_{2}$ drives the oil to well, then the oil is pumped to ground to condensate and storage. The $\mathrm{CO}_{2}$ is separated and pumped to well to use again.

\subsubsection{Process Characteristics}

The RF heating rate is quick through the volumetric heating mode or selective heating mode. The process can be controlled automatically and all the heating process will not pollute environment. Development prospect of $\mathrm{RF} / \mathrm{CF}$ technology is bright due to the advantages as following:

- High oil recovery rate. By using RF/CF technology, 4 5 units power is produced by consuming 1 unit power. It has higher economic benefits than ICP technology (In-situ Retorting Technology), which produces 3.5 units power by consuming 1 unit power.

- The oil industry equipment is used to drill oil well in oil shale. RF antenna or transmitter is sent down to the underground and emits ray to heat the oil shale.

- Crude oil is extracted and driven to well by injecting critical $\mathrm{CO}_{2}$.

- $\mathrm{CO}_{2}$ is separated and reinjected into well to recycle using. At the same time, oil and gas can be refined to gasoline, fuel oil and other products.

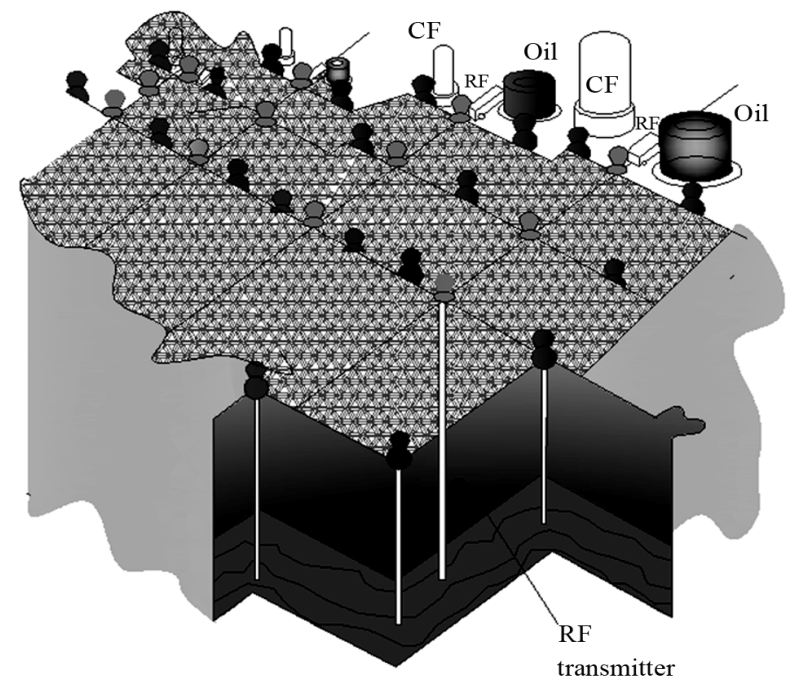

Figure 3. Process of RF/CF technology.
- Oil and gas can be extracted in only few months, it is quicker that the other in-situ technology, which may need many years.

- The heating power can be adjust easily, and will provide a great variety of the products.

- This technology can be used in the extraction of shale oil, sand oil and heavy oil, and it has no residual and will not pollute the groundwater.

- Selective heating mode. It will heat the specified field quickly to the aim temperature.

However, amounts of greenhouse gas will be inevitably produced in the process of shale oil extraction, such as $\mathrm{CO}_{2}$. Raytheon has done a lot of work in the field of $\mathrm{CO}_{2}$ isolation and made some achievements. At present Raytheon has obtained the patent of $\mathrm{CO}_{2}$ isolation technology for RF/CF process $[10,11]$. For the traditional isolation technology, the isolation effects will affected by permeability, storage capacity, space size, temperature and so on. RF/CF technology has avoided these problems by using a closed injection-isolation system. The extraction mode will not discharge any $\mathrm{CO}_{2}$. The RF/CF technology needs lots of energy to get the radio frequency weave, electric power is the original choice, but it is expensive, so Raytheon has began to research the using of alternative sources, such wind energy and solar energy. The research work will build a firm foundation for the RF/CF technology application in the extraction of shale oil.

\subsection{RF Technology Introduction of LLNL}

The RF heating technology has been researched by America Illinois Institute of Technology in the late of 1970s. Vertical combination electrode was used to heat the shale slowly. It needs lots of time to diffuse the heat by conduction. To avoid this defect, LLNL used radio frequency to heat the shale. The RF has a strong penetrating power and can be controlled easily (see Figure 4).

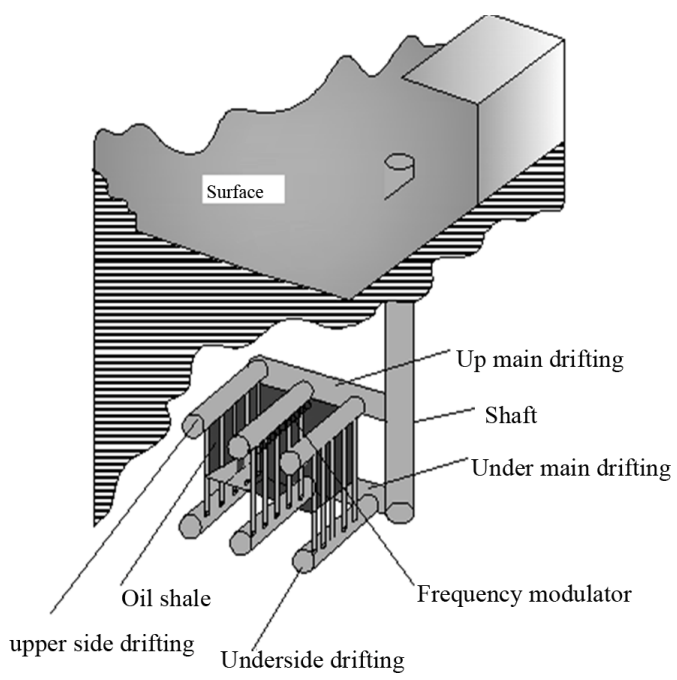

Figure 4. Process of LLNL technology. 


\section{Conclusions}

In a word, the reserve of oil shale is abundant and its distribution is concentrated, maybe it will become the succession energy. The RF technology plays a main pole in the research of shale oil extraction, and has a bright application future. The bottleneck problem for RF development is the power consumption. Because a lot of electric power is used to get microwave, the cost of technology applying is higher. Generally, it needs one microwave generator for every heating well, but it will increase the cost also. The location of generator in well is important. It is still a question now for how to locate the generator, in order to get the longest effective distance and highest utilization efficiency.

The shale oil and gas is a good fuel for boiler, and it can be used to generate electricity. It is a circulation process to use one part of shale oil to generate electricity and then to generate microwave. How to build the circulation in shale oil extraction is a new task.

\section{REFERENCES}

[1] R. J. Li and D. L. Xie, "Research and Development Technologies of Novel Oil Shale Utilization," Modem Chemical Industry, Vol. 30, No. 5, 2010, pp.16-20.

[2] J. R. Dyni, "Geology and Resources of Some World Oil Shale Deposit,” Oil Shale, Vol. 20, No. 3, 2003, pp. 193-
252.

[3] C. F. Knutson, "Developments in Oil Shale in 1989," AAPG Bulletin, Vol. 74, 1990, pp. 372-379

[4] C. Yan and X. M. Jiang, "The Utilization Research of Oil Shale Resources in China,” China Resource, No. 9, 2000, pp. 22-26.

[5] D. X. Liu, H. Y. Wang, D. W. Zheng, C. H. Fang and Z. X. Ge, "Research of In-Situ Technology in the World Oil Shale Extraction,” Nature Gas Industry, Vol. 29, No. 5, 2009, pp. 128-132.

[6] K. D. Savag, "Method of Heating an Oil Shale Formation,” US Patent No. 4524826, 1985.

[7] H. L. Liu, D. X. Liu, C. H. Fang, H. Q. Xue and H. Y. Wang, "Microwave Heating Technology of In-Situ Oil Shale Developing,” Acta Petraolei Sinica, Vol. 31, No. 4, 2010, pp. 623-625.

[8] H. J. Heat, "Sources with Conductive Material for In-Situ Thermal Processing of an Oil Shale Formation,” US Patent No. 6929067, 2005.

[9] F. G. Wen and G. F. Zhu, "The Development of World Oil Shale Production Technology,” Foreign Oilfield Engineering, Vol. 25, No. 1, 2009, pp. 1-5.

[10] X. W. Feng, C. Chen and D. Y. Chen, "Research of InSitu Technology in Oil Shale Extraction,” China Mining, Vol. 20, No. 6, 2011, pp. 84-87.

[11] K. Hatfield, R. Coates and D. Smoot, "Method for Recovery of Hydrocarbon Oils from Oil Shale and Other Carbonaceous Solids,” US Patent No. 0202985 A1, 2008. 